\title{
Binary Organic Nanoparticles with Bright Aggregation- Induced Emission for Three-Photon Brain Vascular Imaging
}

\author{
Maixian Liu ${ }^{\mathrm{a}, \mathrm{b}}$, Bobo $\mathrm{Gu}^{\mathrm{c} \ddagger}$, Wenbo Wu ${ }^{\mathrm{d} \ddagger}$, Yukun Duan ${ }^{\mathrm{d}}$, Hongji Liu ${ }^{\mathrm{a}}$, Xiangquan Deng ${ }^{\mathrm{a}}$, \\ Miaozhuang Fan ${ }^{\mathrm{a}, \mathrm{b}}$, Xiaomei Wang ${ }^{\mathrm{e}}$, Xunbin Wei, ${ }^{\mathrm{c}, \text {, Ken-Tye Yong}}{ }^{\mathrm{g}}$, Ke Wang ${ }^{\text {a* }}$, Gaixia \\ $\mathrm{Xu}^{\mathrm{b} *}$ and Bin Liu ${ }^{\mathrm{d} *}$ \\ ${ }^{a}$ Key Laboratory of Optoelectronics Devices and Systems of Ministry of Education and Guangdong Province, \\ College of Optoelectronic Engineering, Shenzhen University, Shenzhen 518060, China \\ ${ }^{\mathrm{b}}$ National-Regional Key Technology Engineering Laboratory for Medical Ultrasound, Guangdong Key Laboratory \\ for Biomedical Measurements and Ultrasound Imaging, Department of Biomedical Engineering, School of \\ Medicine, Shenzhen University, Shenzhen, China, 518055
}

${ }^{c}$ Med-X Research Institute \& School of Biomedical Engineering, Shanghai Jiao Tong University, 1954 Huashan Road, Shanghai 200030, China

${ }^{\mathrm{d}}$ Department of Chemical and Biomolecular Engineering, National University of Singapore, 4 Science Drive 4, Singapore 117585, Singapore

${ }^{\mathrm{e}}$ Department of Physiology, School of Basic Medical Sciences, Shenzhen University, Shenzhen, China, 518055

${ }_{\mathrm{f}}^{\mathrm{f}}$ Beijing Advanced Innovation Center for Biomedical Engineering, Beihang University, Beijing, 100083, China

${ }^{\mathrm{g}}$ School of Electrical and Electronic Engineering, Nanyang Technological University, 50 Nanyang Avenue, Singapore 639798, Singapore

$\ddagger$ These authors contributed equally. 


\section{Materials and Methods}

\section{Materials}

All chemicals were used without further purification. 1,1,2,2-Tetrakis(4-bromophenyl)ethylene (TPE-Br) was purchased from Sigma-Aldrich. Bovine serum albumin (BSA, fatty acid free) was obtained from Yancheng Saibao Biotech. co. ltd. TBDTT has been prepared in our previous work. ${ }^{1}$

\section{Formulation of organic nanoparticle and BONAP}

Before synthesizing BONAPs, stock solution of TPE-Br (2 mM or $10 \mathrm{mM})$ in THF and of TBDTT $1 \mathrm{mM}$ in THF were prepared. For molar ratios TPE-Br:TBDTT $=0,3,6,12,20 \mu \mathrm{L}$ of TBDTT and correspondingly $0,30,60,120 \mu \mathrm{L}$ of $2 \mathrm{mM}$ TPE-Br in THF were added into $400 \mu \mathrm{L}$ of DMSO. For molar ratios of TPEBr:TBDTT $=25,50,100,20 \mu \mathrm{L}$ of TBDTT and correspondingly 50, 100, $200 \mu \mathrm{L}$ of $10 \mathrm{mM}$ TPE-Br in THF were added into $400 \mu \mathrm{L}$ of DMSO. The solution was mixed thoroughly before injection into $4 \mathrm{~mL}$ of $10 \mathrm{mg} / \mathrm{mL}$ BSA solution in a $50 \mathrm{~mL}$ centrifuge tube under vortex. The vortex was then kept for $10 \mathrm{~s}$ before further experiments. TBDTT NPs were synthesized using the same method as that of BONAPs. For nanoparticles used in biological experiments, the suspension of BONAP nanoparticle after injection was spin-filtered (MWCO $100 \mathrm{kda}$ ) at least 3 times to rid of excessive DMSO and THF and to buffer-exchange with PBS, followed by sterilization via filtration through $0.2 \mu \mathrm{m}$ pore filter.

\section{Quantum yield measurement}

The quantum yield of TBDTT in BONAP was measured in reference to riboflavin $(\Phi=0.3$ in ethanol, exc: $455 \mathrm{~nm}$ ). Briefly, the PL of NP excited at $455 \mathrm{~nm}$ was measured. and its absorption spectra was taken. The QY of TBDTT in BONAP was calculated by the following equation:

$$
\Phi_{T B D T T}=\Phi_{\text {riboflavin }} \cdot \frac{A_{\text {riboflavin }}}{A_{T B D T T}} \cdot \frac{I_{T B D T T}}{I_{\text {riboflavin }}} \cdot \frac{n_{\text {water }}^{2}}{n_{\text {ethanol }}^{2}}
$$

where, $\Phi$ stands for quantum yield; A for absorbance at $455 \mathrm{~nm}$; I for integrated PL intensities; $\mathrm{n}$ for refractive indices (NP was suspended in water while riboflavin was dissolved in ethanol). All measurements were performed when the PL was in linear relationship to fluorophore concentration to avoid inner filter effect.

\section{Lifetime measurement}

Lifetime of photoluminescence from TBDTT was performed by time-correlated single photon counting (TCSPC) method. TCSPC diode laser at $445 \mathrm{~nm}$ was chosen in order to excite TBDTT only. The measurement was carried out on Edinburgh FS1000 Spectrometer. The average lifetimes of BONAPs at different molecular ratios were calculated according to the following equation:

$$
\tau_{a}=\frac{\sum\left(A_{i} \cdot \tau_{i}^{2}\right)}{\sum A_{i} \cdot \tau_{i}}
$$

$\tau_{\mathrm{a}}$ stands for average lifetime, $\mathrm{A}_{\mathrm{i}}$ for the amplitude of the $\mathrm{i}$ th lifetime component and $\tau_{\mathrm{i}}$ for the respective lifetime. 


\section{Radiative decay rate}

Calculated results from time-resolved decay of photoluminescence at $700 \mathrm{~nm}$. Average lifetimes were obtained by exciting sample with TCSPC diode at $445 \mathrm{~nm}$. Radiative rate was derived from the following equation:

$$
\tau_{r}=\frac{\tau_{f l}}{\Phi_{f l}}, k_{r}=\frac{1}{\tau_{r}}, \Phi_{f l}=\frac{k_{r}}{k_{r}+k_{n r}}
$$

Where, $\tau_{r}$ is radiative lifetime, $\tau_{f l}$ is fluorescent lifetime detected by TCSPC method. $k_{r}$ is radiative rate and $k_{n r}$ is non-radiative rate. $\Phi_{f l}$ is the quantum yield of TBDTT.

\section{Simulation}

Molecular Dynamics (MD) simulations of BONAPs with molecular ratios were performed using Gromacs program suite ${ }^{2}$ and all-atom optimized potentials for liquid simulations (OPLS-AA) force field. The force field and topology files were generated by TPPmktop, and the CM atomic charges were calculated by Gaussian $16{\mathrm{~A} 03^{3} \text { and Multiwfn program }}^{4}$. The aggregation of TBDTT in aqueous solution was simulated in a box of dimensions $100 \times 100 \times 100 \AA^{3}$. 20000 water molecules, 100 TBDTT molecules and TPE-Br molecules of different molecular ratios were packed randomly using packmol program ${ }^{5}$.

The energy minimizing of these molecules was performed firstly under NVT ( $\mathrm{T}=300 \mathrm{~K}$ ) ensemble by steepest descent algorithm, and the systems were then equilibrated under NPT ( $\mathrm{T}=298.15 \mathrm{~K}$ and $\mathrm{P}=1 \mathrm{~atm})$ ensemble during $1 \mathrm{~ns}$ through an annealing from $60 \mathrm{~K}$ to $298.15 \mathrm{~K}$ with the time step of $1 \mathrm{ps}$. Velocityrescale thermostat ${ }^{6}$ was used to control the temperature at $298.15 \mathrm{~K}$. Berendsen's barostat with a isothermal compressibility constant of $4.5 \times 10^{-5}$ was used to control the pressure at $1.01325 \times 10^{5} \mathrm{~Pa}$. The time constants of couplings for temperature and pressure were $0.1 \mathrm{ps}$ and $1.0 \mathrm{ps}$, respectively. Periodic boundary conditions were applied in all three directions to minimize the edge effects in a finite system. Particle-mesh Ewald (PME) method with a cut-off distance of $10 \AA$ was applied to treat the electrostatic interactions and the van der Waals forces. All bond lengths were constrained via the LINCS algorithm. Finally, after the formation of TBDTT nanoparticles during equilibration simulation, $10 \mathrm{~ns}$ production molecular dynamics simulations were conducted to obtain enough configurations for data collection, the trajectory was saved every 1 ps. The further statistics results were calculated from the trajectory data by Python scripts written by ourselves.

\section{In vitro and In vivo toxicity}

BONAP at 25:1 was chosen to test the biocompatibility before applied onto BALB/C. In vitro toxicity was estimated by MTS assay. $5000 \mathrm{HeLa}$ cells were planted on 96-well plate one day before applying BONAP. On the second day, BONAP solution with different concentrations was applied onto HeLa cells with six replicates $(\mathrm{n}=6)$ and incubated at $37{ }^{\circ} \mathrm{C}$ with $5 \% \mathrm{CO}_{2}$ for 24,48 , $72 \mathrm{hrs}$. At each time point, $10 \mathrm{uL}$ of MTS reagent (Promega) was added to each well and the readings at $490 \mathrm{~nm}$ were taken on plate reader 50 mins after MTS reagent was added. 
In vivo toxicity was carried out by using BALB/C mice ( $\mathrm{n}=7$ ), each of which was injected either $50 \mathrm{mg} / \mathrm{mL}$ (TBDDT $2.5 \mathrm{mg} / \mathrm{kg}$ ) NP or PBS (control). Weight changes were tracked for 15 days. On day 15, all mice were put under anesthesia using isofluorane and blood was taken. Major organs were weighed, and organic coefficient was calculated. Blood chemistry indicators were characterized on Mindray chemistry analyzer. H\&E stain of major organ slices were performed after fixing them in $10 \%$ formalin for 2 days.

\section{Measurement of three-photon excitation cross-section ( $\left.\eta \sigma_{3}\right)$}

The details of measuring $\eta \sigma_{3}$ are introduced in previous article ${ }^{7}$. Briefly, $\eta \sigma_{3}$ at $1680 \mathrm{~nm}$ for sulforhodamine 101 (SR101) was used as a reference. $\eta \sigma_{3}$ excited at other wavelengths from fluorescent labels was referenced to this value. Assuming equal molar concentrations, the resultant $\eta \sigma_{3, \lambda}=\eta \sigma_{3,1680} \mathrm{P}_{1680}{ }^{3} \tau_{\lambda}{ }^{2} \mathrm{~S}_{\lambda} /\left(\mathrm{P}_{\lambda}{ }^{3} \tau_{1680}{ }^{2} \mathrm{~S}_{1680}\right)(\lambda / 1680)^{3}$, where $\eta \sigma_{3, \lambda}$ is the wavelength dependent 3photon action cross section, $\eta \sigma_{3,1680}=65.5 \times 10^{-84} \mathrm{~cm}^{6}(\mathrm{~s} / \mathrm{photon})^{2}$ is $\eta \sigma_{3}$ for SR101 excited at 1680 $\mathrm{nm}, \mathrm{P}_{1680}$ and $\mathrm{P}_{\lambda}$ are the measured excitation power on the sample, $\tau_{1680}$ and $\tau_{\lambda}$ are the measured pulse widths on the sample, and $S_{1680}$ and $S_{\lambda}$ are the measured 3-photon fluorescence signals, for excitation at $1680 \mathrm{~nm}$ and other wavelengths, respectively.

The $\eta \sigma_{3}$ measurement system comprises two parts: a tunable femtosecond laser source (tunable from $1600 \mathrm{~nm}$ to $1840 \mathrm{~nm}$ ) and a fluorescence signal generation/detection part. Soliton selffrequency shift (SSFS) in 8.5-m large-mode-area (LMA) fiber (LMA-PM-35, NKT Photonics) was used to generate tunable femtosecond excitation pulses, pumped by a $1-\mathrm{MHz} 1550-\mathrm{nm}$ fiber laser (FLCPA-02CSZU, Calmar). A $965 \mathrm{~nm}$ dichroic (T965lpxxrxt-UF1, Chroma) was used to transmit the excitation pulses and to reflect the fluorescence signal to detectors. A $0.45 \mathrm{NA}$ air objective lens (A Plan 20×/0.45 NA, Zeiss) was used to focus the excitation light and epi-collect the fluorescence signals. A GaAs photomultiplier tube (PMT, H7422p-50, Hamamatsu) was used for fluorescence signal detection. The current signal from the PMT was converted to voltage and amplified in a wide bandwidth amplifier (C6438-01, Hamamatsu), and then fed into a photon counter (SR400, SRS) for photon counting.

\section{Three photon-imaging of mouse vascular structure}

For 3PM, dual-channel detection was performed to record 3-photon fluorescence and THG signals simultaneously, using a multiphoton microscope (MOM, Sutter), excited by an energetic femtosecond source operating at the 1700-nm window. A GaAsP PMT (H7422p-40, Hamamatsu) and a GaAs PMT (H7422p-50, Hamamatsu) were used for fluorescent and THG signal detection, respectively. In front of the GaAsP PMT, a 630/92 band-pass filter (FF01-630/92-25, Semrock) and a 593 long-pass filter (FF01593/LP-25, Semrock) were used, while in front of the GaAs PMT, a 558/20 band-pass filter (FF01-558/2025, Semrock) was used. Image acquisition and processing were performed using ScanImage (Vidrio Technologies) and ImageJ (NIH), respectively. 

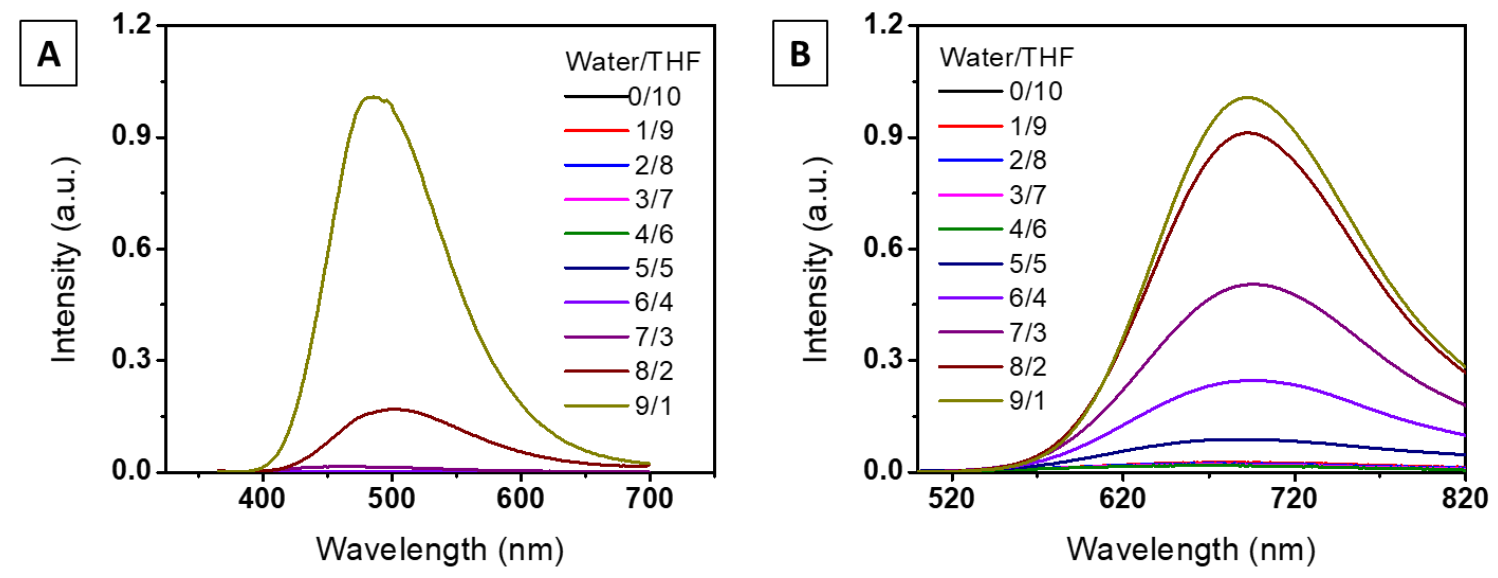

Figure S1. A) PL spectra of TPE-Br in THF and Water/THF mixture at different volume ratios, $\lambda_{\mathrm{exc}}=355$ $\mathrm{nm}$, [TPE-Br] $=20 \mu \mathrm{M}$. B) PL spectra of TBDTT in THF and Water/THF mixture at different volume ratios, $\lambda_{\text {exc }}=455 \mathrm{~nm},[$ TBDTT $]=10 \mu \mathrm{M}$.

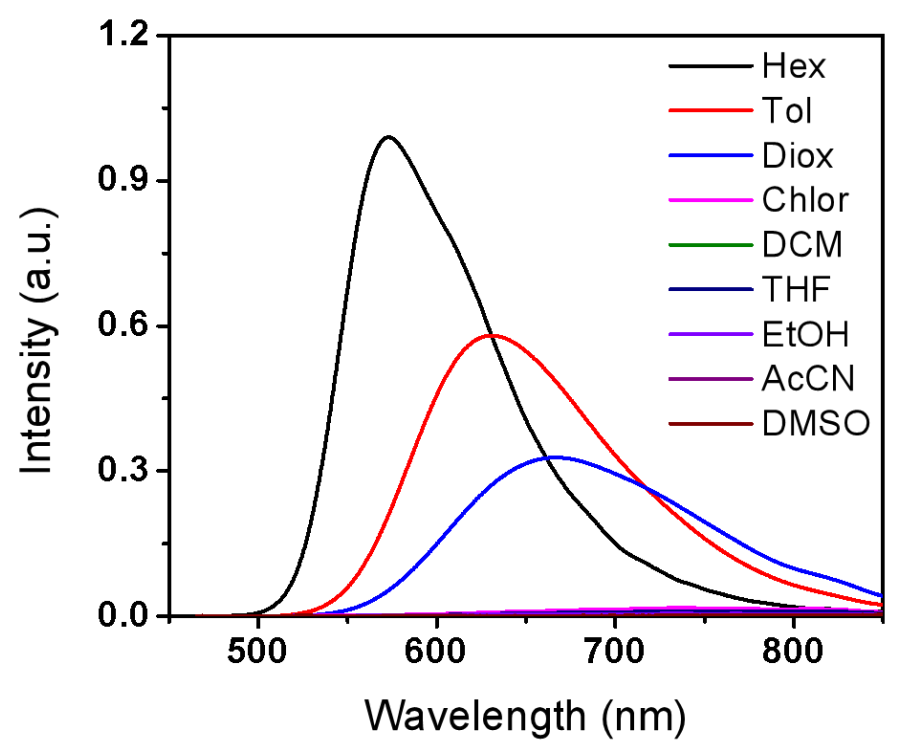

Figure S2. Photoluminescence spectra of TBDTT in different solvents. [TBDTT] $=3.0 \mu \mathrm{M}, \lambda_{\mathrm{exc}}=455 \mathrm{~nm}$. Abbreviations in the legend: Hex-Hexane; Tol-Toluene; Diox-1,4-Dioxane; Chlor-Chloroform; DCMDichloromethane; THF-Tetrahydrofuran; EtOH-Ethanol; AcCN-Acetonitrile; DMSO-Dimethylsulfoxide. 

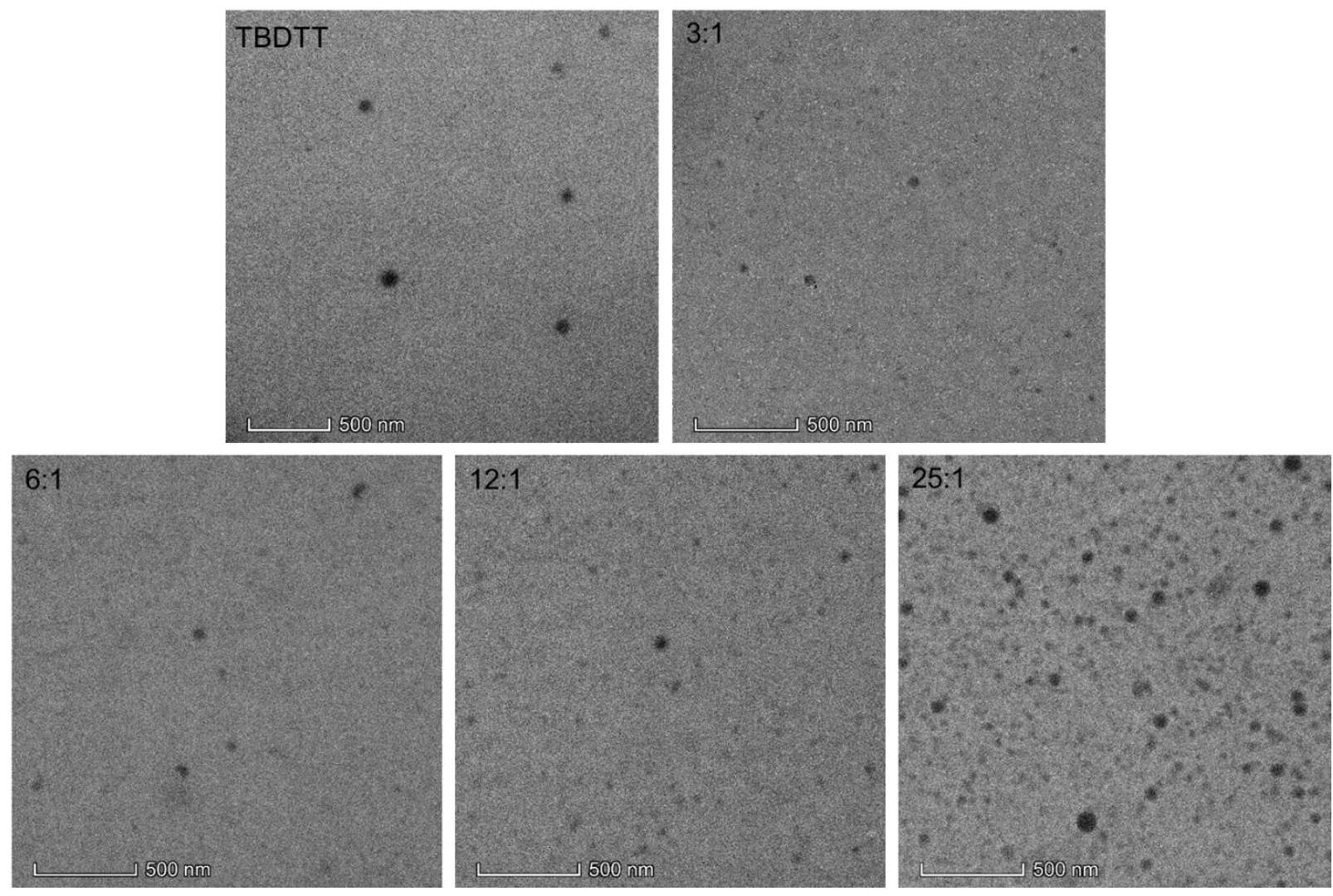

Figure S3. Transmission electron microscopy images of BONAPs with different molar ratios between TPE-Br and TBDTT.

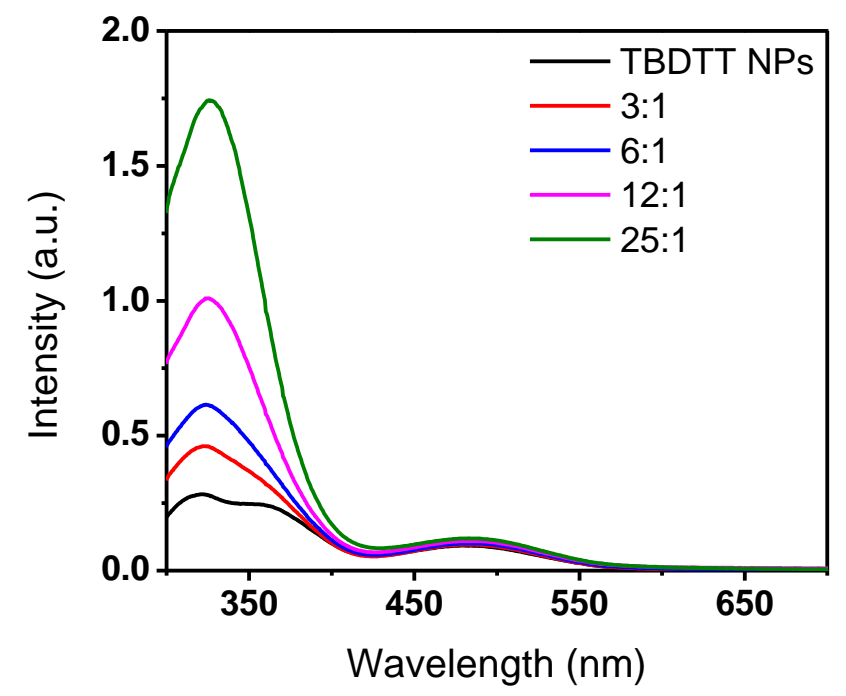

Figure S4. Absorption of BONAPs at TPE-Br:TBDTT molar ratios from 0 to 25:1. BONAP concentration is represented by that of [TBDTT], and [TBDTT] was kept at $5.0 \mu \mathrm{M}$ for all ratios. 

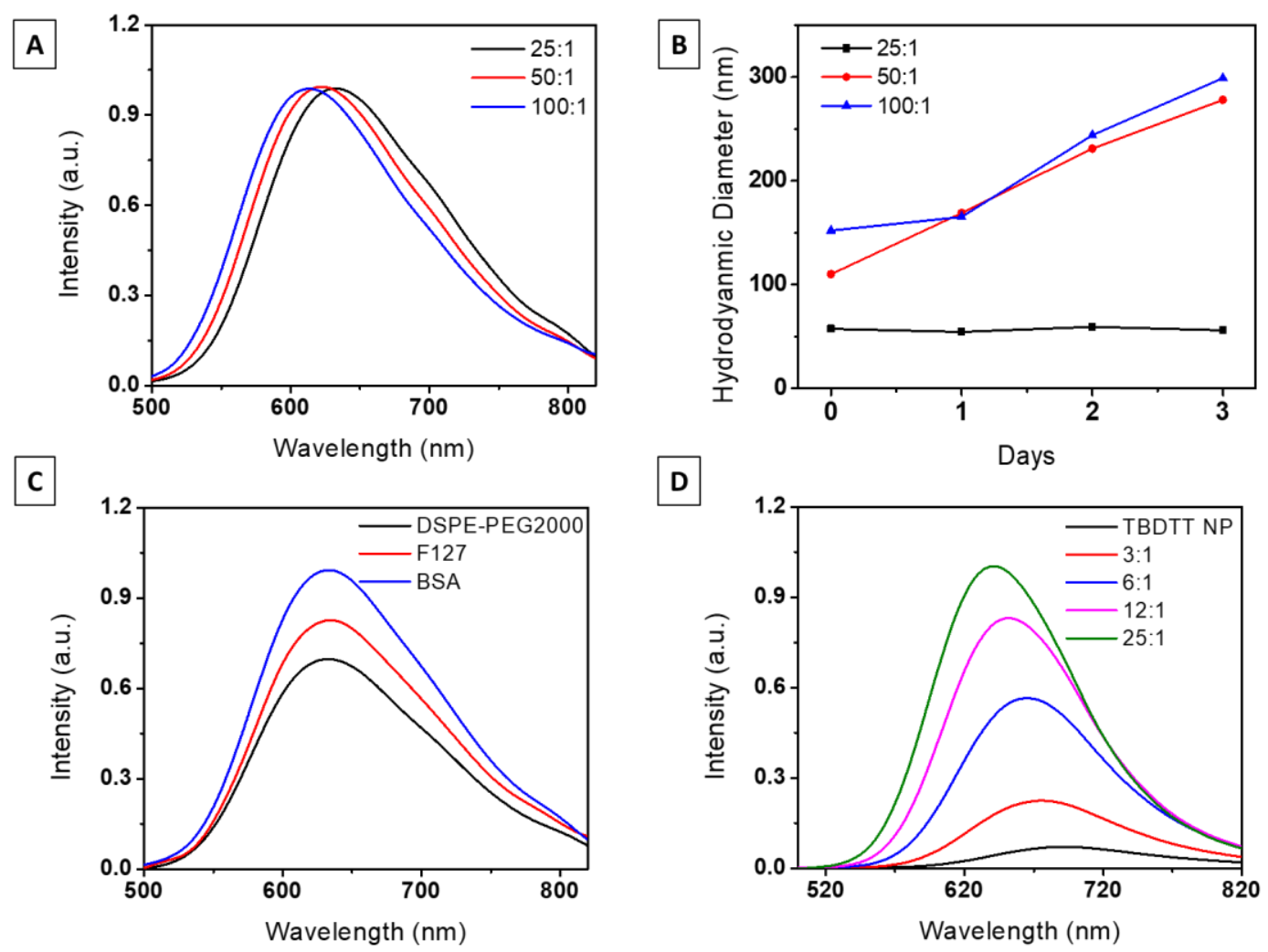

Figure S5. A) PL of BONAPs synthesized 25:1, 50:1, 100:1. B) Hydrodynamic size of BONAP of 25:1, 50:1 and 100:1. Hydrodynamic sizes were measured by dynamic light scattering (DLS) immediately after synthesis ( 0 day), 1, 2 and 3 days. C) Comparison of PL spectra of BONAP (TPE-Br:TBDTT = 25:1) with different matrices. D) PL spectra of BONAPs without BSA as protecting matrix. [TBDTT] $=5.0 \mu \mathrm{M}, \lambda_{\mathrm{exc}}$ $=455 \mathrm{~nm}$.

Table S1 Lifetime measurement of BONAPs. TCSPC diode at $445 \mathrm{~nm}$ was used to excite TBDTT inside BONAP only. $A_{i}(i=1,2,3)$ stands for amplitude of each lifetime assignment in each sample measurement.

\begin{tabular}{|c|c|c|c|c|}
\hline $\begin{array}{c}\text { Sample } \\
\text { Name }\end{array}$ & $\boldsymbol{\tau}_{\mathbf{1}}(\mathbf{n s}) / \mathbf{A}_{\mathbf{1}}$ & $\boldsymbol{\tau}_{\mathbf{2}}(\mathbf{n s}) / \mathbf{A}_{\mathbf{2}}$ & $\boldsymbol{\tau}_{\mathbf{3}}(\mathbf{n s}) / \mathbf{A}_{\mathbf{3}}$ & $\begin{array}{c}\text { Average } \\
\text { Lifetime }\left(\boldsymbol{\tau}_{\mathbf{a}}\right. \\
\mathbf{n s})\end{array}$ \\
\hline $\begin{array}{c}\text { TBDTT } \\
\text { NPs }\end{array}$ & $1.02 / 334.875$ & $4.88 / 503.69$ & $18.74 / 133.69$ & 11.18 \\
\hline $3: 1$ & $1.35 / 271.53$ & $5.20 / 584.2$ & $15.01 / 148.21$ & 8.83 \\
\hline $6: 1$ & $1.38 / 166.43$ & $5.41 / 653.12$ & $13.83 / 159.31$ & 8.36 \\
\hline $12: 1$ & $5.65 / 788.78$ & $12.79 / 193.48$ & N/A & 8.20 \\
\hline $25: 1$ & $6.76 / 805.87$ & $13.54 / 106.97$ & N/A & 8.188 \\
\hline
\end{tabular}



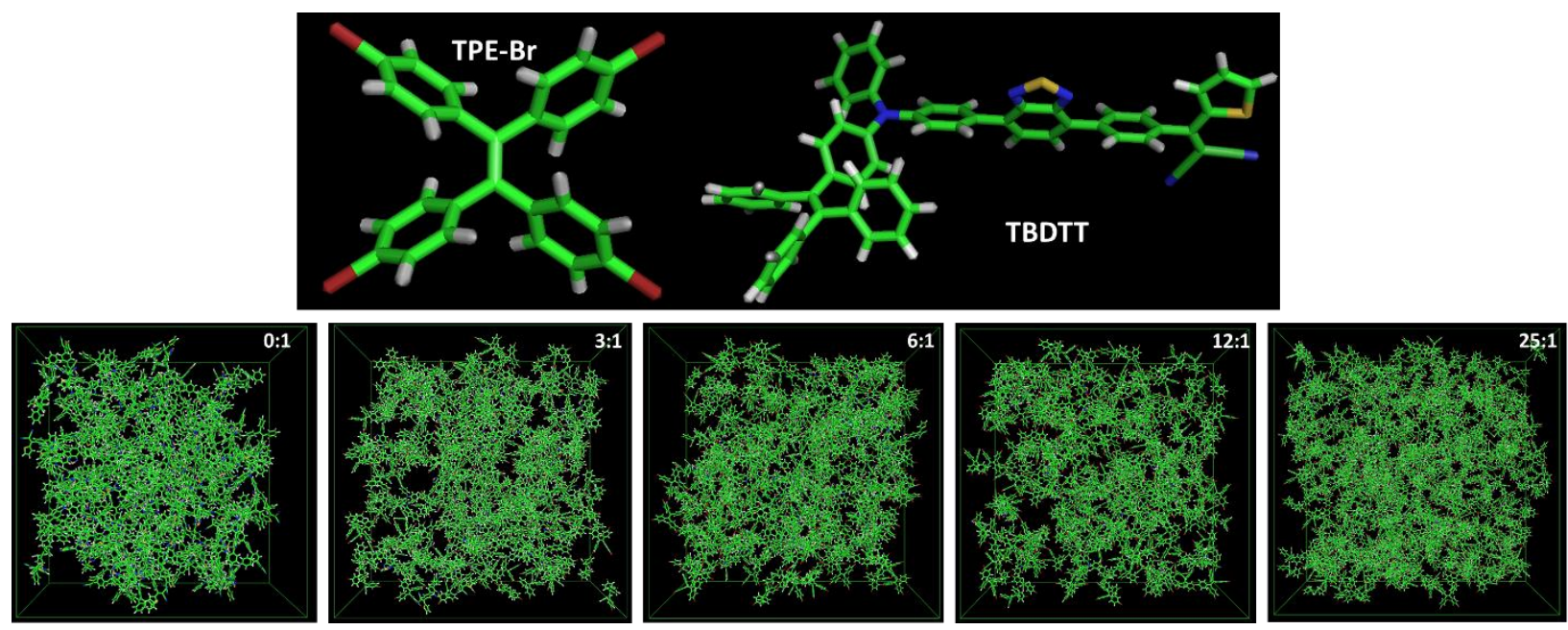

Figure S6. BONAPs formation simulation. The optimized configuration of TPE-Br and that of TBDTT are presented in stick form in the first line. The simulation box of each BONAP at indicated ratios are presented with both TPE-Br and TBDTT in the second line.

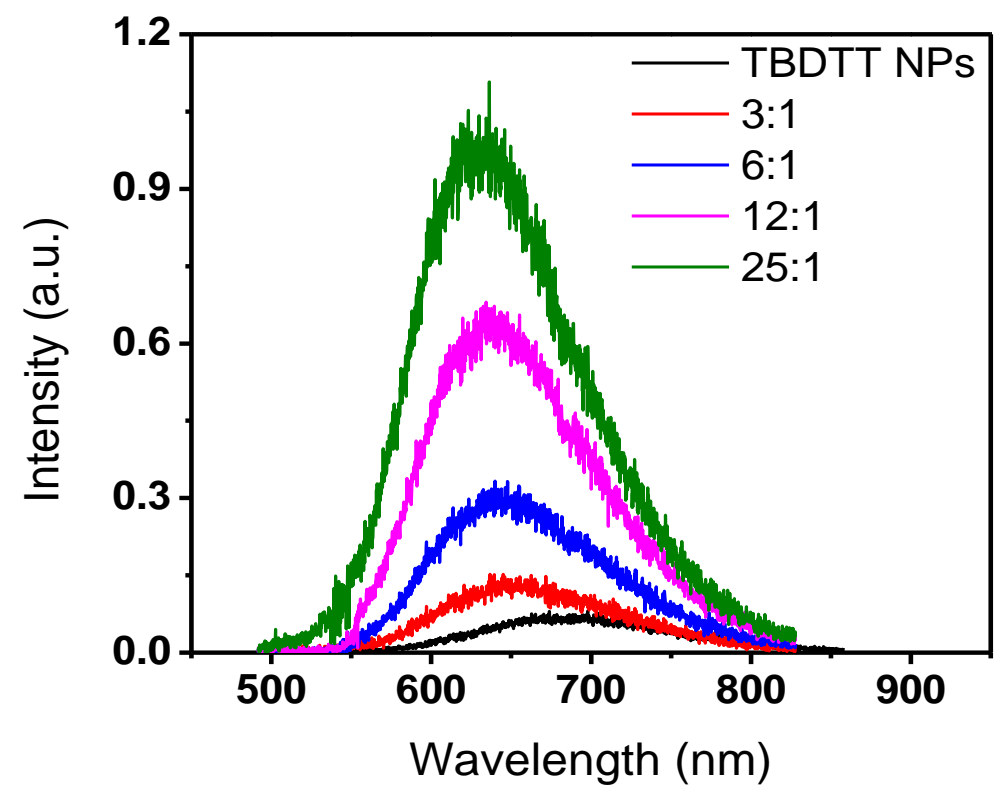

Figure S7. 3PF spectra of BONAPs with different molecular ratio of TPE-Br to TBDTT. [TBDTT] $=100 \mathrm{uM}, \lambda_{\mathrm{exc}}=1610 \mathrm{~nm}$. 


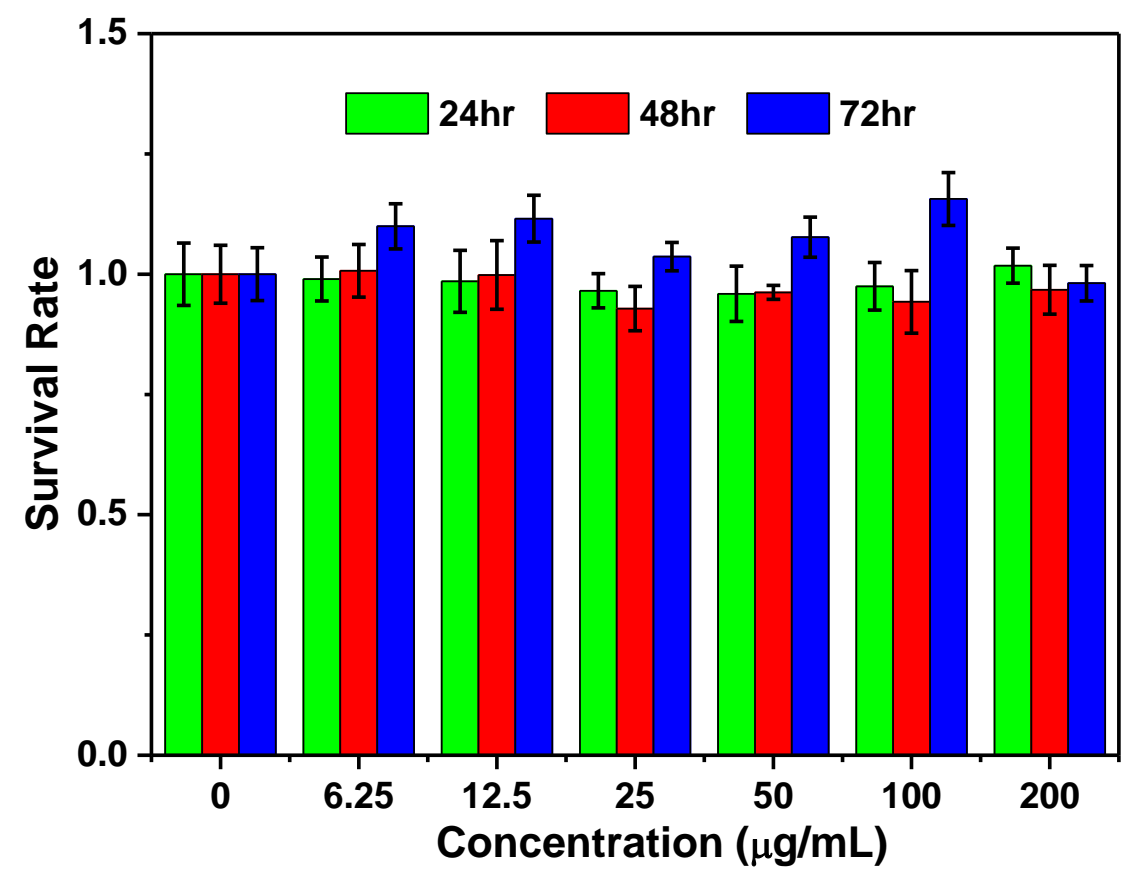

Figure S8. Survival rate of HeLa cells upon incubation with BONAPs (TPE-Br to TBDTT molar ratio of 25:1). MTS assay of BONAPs unto HeLa cells with different concentrations. The detection was performed in 3 consecutive days. 

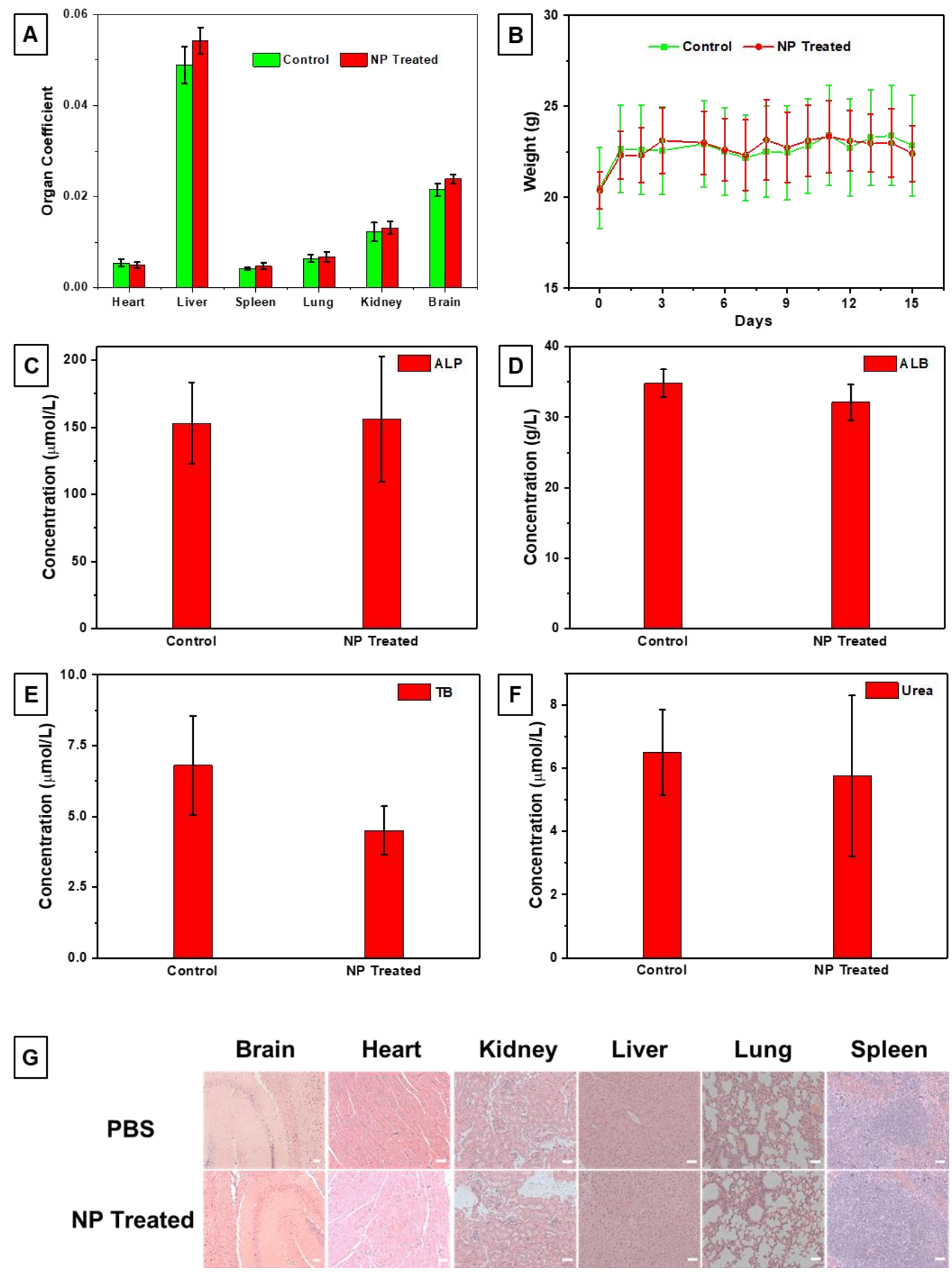

Figure S9. Evaluation of toxicity in vivo. BALB/C mice of 8 weeks old were treated with NP or PBS. A) Organ Coefficient and B) Weight Tracking shows no statistical difference between NP- or PBS-treated group. C-F) Important blood chemical indicators. G) Major organ slices stained with H\&E. Scale bar in each picture is $50 \mu \mathrm{m}$. 
A

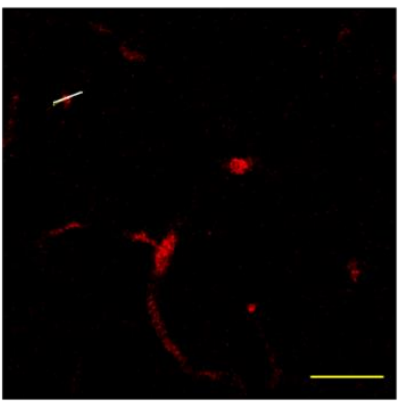

B

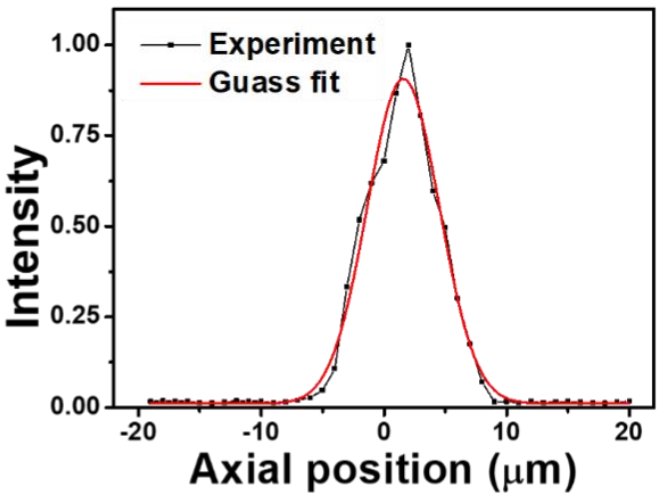

C

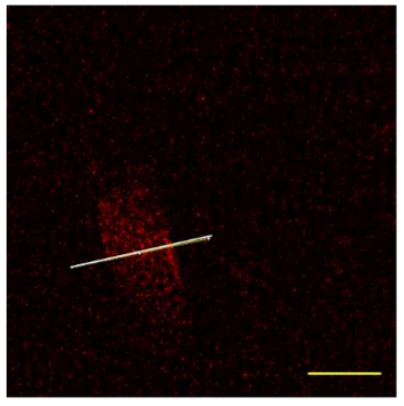

D

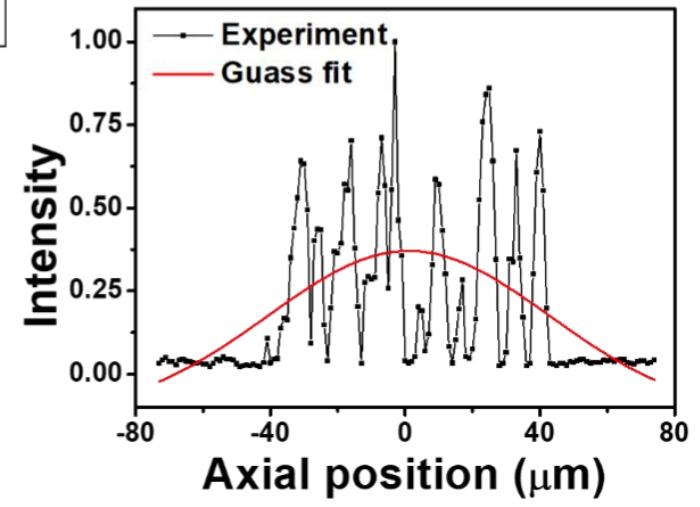

Figure S10. A, C) Vascular structure from Figure 6 D, E at $1200 \mu \mathrm{m}(\mathrm{A})$ and $1680 \mu \mathrm{m}(\mathrm{C})$ with measured blood vessels marked by white lines. B, D) 3PEF signal and background signal across the white lines in A and $\mathrm{C}$, respectively. Scale Bar (yellow): $50 \mu \mathrm{m}$. 

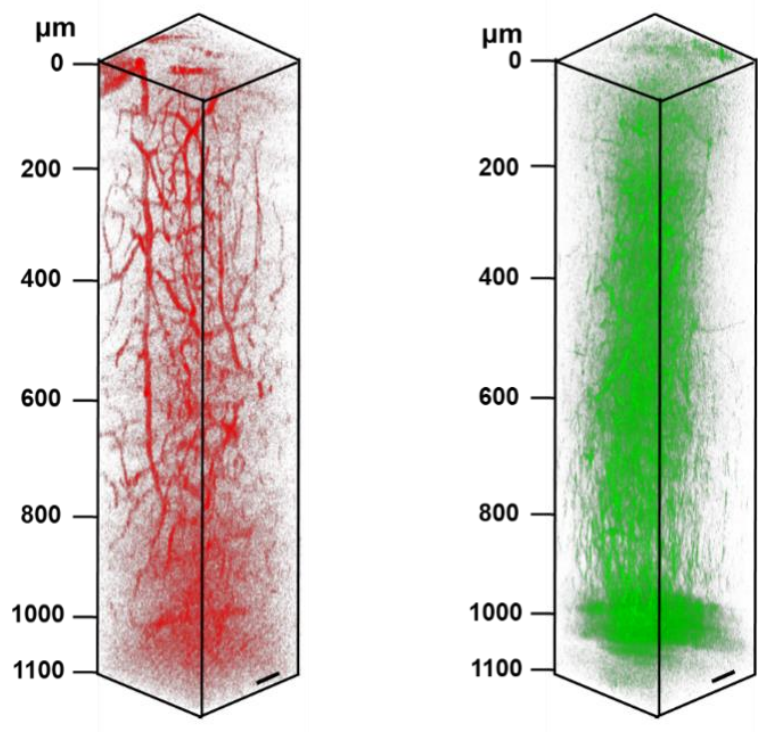

Figure S11. 3PF imaging of an 8-week old BALB/C mouse injected with $2.5 \mathrm{mg} / \mathrm{kg}$ TBDTT NP, $\lambda_{\mathrm{exc}}=$ $1610 \mathrm{~nm}$. Left: 3-photon fluorescence imaging of mouse brain vascular structure; Right: Third-harmonic generation (THG) imaging of mouse brain structure. Scale Bar: $50 \mu \mathrm{m}$

\section{References:}

1. Wu, M.; Wu, W.; Duan, Y.; Li, X.; Qi, G.; Liu, B., Photosensitizer-Bacteria Biohybrids Promote Photodynamic Cancer Cell Ablation and Intracellular Protein Delivery. Chem. Mater. 2019, 31, 72127220 .

2. Abraham, M. J.; Murtola, T.; Schulz, R.; Páll, S.; Smith, J. C.; Hess, B.; Lindahl, E., GROMACS: High Performance Molecular Simulations through Multi-Level Parallelism from Laptops to Supercomputers. SoftwareX 2015, 1-2, 19-25.

3. Frisch, M. J.; Trucks, G. W.; Schlegel, H. B.; Scuseria, G. E.; Robb, M. A.; Cheeseman, J. R.; Scalmani, G.; Barone, V.; Petersson, G. A.; Nakatsuji, H.; Li, X.; Caricato, M.; Marenich, A. V.; Bloino, J.; Janesko, B. G.; Gomperts, R.; Mennucci, B.; Hratchian, H. P.; Ortiz, J. V.; Izmaylov, A. F.;

Sonnenberg, J. L.; Williams; Ding, F.; Lipparini, F.; Egidi, F.; Goings, J.; Peng, B.; Petrone, A.; Henderson, T.; Ranasinghe, D.; Zakrzewski, V. G.; Gao, J.; Rega, N.; Zheng, G.; Liang, W.; Hada, M.; Ehara, M.; Toyota, K.; Fukuda, R.; Hasegawa, J.; Ishida, M.; Nakajima, T.; Honda, Y.; Kitao, O.; Nakai, H.; Vreven, T.; Throssell, K.; Montgomery Jr., J. A.; Peralta, J. E.; Ogliaro, F.; Bearpark, M. J.; Heyd, J. J.; Brothers, E. N.; Kudin, K. N.; Staroverov, V. N.; Keith, T. A.; Kobayashi, R.; Normand, J.; Raghavachari, K.; Rendell, A. P.; Burant, J. C.; Iyengar, S. S.; Tomasi, J.; Cossi, M.; Millam, J. M.; Klene, M.; Adamo, C.; Cammi, R.; Ochterski, J. W.; Martin, R. L.; Morokuma, K.; Farkas, O.; Foresman, J. B.; Fox, D. J. Gaussian 16 Rev. A.03, Wallingford, CT, 2016.

4. Lu, T.; Chen, F., Multiwfn: A Multifunctional Wavefunction Analyzer. J. Comput. Chem. 2012, 33, 580-592.

5. Martínez, L.; Andrade, R.; Birgin, E. G.; Martínez, J. M., PACKMOL: A Package for Building Initial Configurations for Molecular Dynamics Simulations. J. Comput. Chem. 2009, 30, 2157-2164.

6. Bussi, G.; Donadio, D.; Parrinello, M., Canonical Sampling through Velocity Rescaling. J. Chem. Phys. 2007, 126, 014101. 
7. Liu, H.; Deng, X.; Tong, S.; He, C.; Cheng, H.; Zhuang, Z.; Gan, M.; Li, J.; Xie, W.; Qiu, P.; Wang, K., In Vivo Deep-Brain Structural and Hemodynamic Multiphoton Microscopy Enabled by Quantum Dots. Nano Lett. 2019, 19, 5260-5265. 